P. Pflug and W. Zwonek

Nagoya Math. J.

Vol. 180 (2005), 121-133

\title{
BERGMAN COMPLETENESS OF UNBOUNDED HARTOGS DOMAINS
}

\author{
PETER PFLUG AND WŁODZIMIERZ ZWONEK
}

\begin{abstract}
Some results for the Bergman functions in unbounded domains are shown. In particular, a class of unbounded Hartogs domains, which are Bergman complete and Bergman exhaustive, is given.
\end{abstract}

For an open set $\Omega \subset \mathbb{C}^{n}$ let us recall that the Bergman kernel is given by the formula

$$
K_{\Omega}(z):=\sup \left\{|f(z)|^{2}: f \in L_{h}^{2}(\Omega),\|f\|_{\Omega} \leq 1\right\}, \quad z \in \Omega,
$$

where $L_{h}^{2}(\Omega)$ denotes the Hilbert space of square integrable holomorphic functions on $\Omega$ and $\|\cdot\|_{\Omega}$ denotes the $L^{2}$ norm on $\Omega$.

Recall that if $K_{\Omega}(z)>0$ for any $z \in \Omega$ then one may define the positive semidefinite Bergman pseudometric $\beta_{\Omega}$

$$
\beta_{\Omega}(z ; X):=\sqrt{\sum_{j, k=1}^{n} \frac{\partial^{2} \log K_{\Omega}(z)}{\partial z_{j} \partial \bar{z}_{k}} X_{j} \bar{X}_{k}}, \quad z \in \Omega, X \in \mathbb{C}^{n} .
$$

The pseudometric $\beta_{\Omega}$ induces the Bergman pseudodistance $b_{\Omega}$ on $\Omega$. If for any $z \in \Omega$ and any $X \in \mathbb{C}^{n} \backslash\{0\}$ there exists an $f \in L_{h}^{2}(\Omega)$ with $f^{\prime}(z) X \neq 0$, then, in fact, $\beta_{\Omega}$ is a metric and $b_{\Omega}$ is a distance. In particular, $\left(\Omega, b_{\Omega}\right)$ is a metric space. For basic properties of Bergman functions see e.g. [Jar-Pfl 1993].

We call the domain $\Omega$ Bergman complete if $b_{\Omega}$ is a distance and the metric space $\left(\Omega, b_{\Omega}\right)$ is complete. There is a number of results on Bergman completeness. We recall only the result of Bremerman (see [Bre 1955]) which states that any Bergman complete domain is pseudoconvex and a recent result of Błocki-Pflug and Herbort (see [Bło-Pfl 1998], [Her 1999]) stating

Received October 1, 2004.

2000 Mathematics Subject Classification: Primary 32A07, 32F45, 32A25; Secondary $32 \mathrm{U} 35,30 \mathrm{C} 85$.

The research was supported by DFG Project No. 227/8-1. 
that any bounded hyperconvex domain is Bergman complete - the bounded domain $D \subset \mathbb{C}^{n}$ is called hyperconvex if there is a negative, plurisubharmonic exhaustion function for the domain D. See also [Chen 2004] for a generalization for the unbounded case.

The most powerful tool used in the proofs of Bergman completeness of domains is the Kobayashi criterion (see [Kob 1962]).

Theorem. (Kobayashi criterion) Let $D$ be a domain in $\mathbb{C}^{n}$ with a Bergman metric. Let $\mathcal{E}$ be a dense subset of $L_{h}^{2}(D)$ such that for any $f \in$ $\mathcal{E}$ and for any sequence $\left(z^{\nu}\right) \subset D$ without accumulation points in $D$ the convergence

$$
\lim _{\nu \rightarrow \infty} \frac{\left|f\left(z^{\nu}\right)\right|^{2}}{K_{D}\left(z^{\nu}\right)}=0
$$

holds. Then $D$ is Bergman complete.

Most of the results on Bergman completeness of domains is restricted to bounded ones. However, recently some papers appeared where the problem of Bergman completeness of unbounded domains was studied (see [Chen 2004], [Chen-Zhang 2002], [Juc 2004], [Chen-Kam-Ohs 2004], [ChenZhang 2004]). Motivated by examples from the last two papers we present a new class of unbounded domains admitting the Bergman metric, which are Bergman exhaustive (recall that the domain $D$ is Bergman exhaustive if for any sequence $\left(z^{\nu}\right) \subset D$ without accumulation points in $D$ the convergence $\lim _{\nu \rightarrow \infty} K_{D}\left(z^{\nu}\right)=\infty$ holds) and Bergman complete (see Theorem 6). The examples we deliver are Hartogs domains with the base equal to $\mathbb{C}^{n}$. Let us recall that in the unbounded case we have to overcome additional, in comparison to the bounded one, difficulties. Namely, positivity of the Bergman kernel $K_{D}$ and the existence of the Bergman metric; they are not automatic and must be proven.

We also apply recent results relating the logarithmic capacity and the Bergman function (see [Zwo 2002], [Pfl-Zwo 2003]) to obtain some results on Bergman exhaustiveness of bounded domains (see Theorem 4). For definition and basic properties of the logarithmic capacity we refer the interested Reader to the book [Ran 1995].

Since the research on various properties of Bergman functions has been concentrated on bounded domains many results concerning these functions were formulated mostly for bounded domains. Therefore, we need to repeat to prove some of the results for the unbounded domains. The proofs of the 
results, which include Theorems 1, 2 and 3, are mostly simple transformations of the appropriate results for bounded domains.

Now for an open set $\Omega \subset \mathbb{C}^{n}$ recall the notion of the pluricomplex Green function with the logarithmic pole at $p \in \Omega$

$$
g_{\Omega}(p, z):=\sup \{u(z)\}, \quad z \in \Omega,
$$

where the supremum is taken over all $u \in \operatorname{PSH}(\Omega)$ (PSH stands here for plurisubharmonic), $u<0$ and $u(\cdot)-\log \|\cdot-p\|$ is bounded from above near $p$. For basic properties of the pluricomplex Green function we refer the Reader to [Kli 1991].

For an open set $\Omega \subset \mathbb{C}^{n}, p \in \Omega, a>0$ denote

$$
A(p, \Omega, a):=\left\{z \in \Omega: g_{\Omega}(p, z) \leq-a\right\}
$$

For $k=0,1, \ldots$, and $X \in \mathbb{C}^{n}$ denote also

$$
K_{\Omega}^{(k)}(p ; X):=\sup \left\{\left|f^{(k)}(p) X\right|^{2}: f \in L_{h}^{2}(D):\|f\|_{\Omega} \leq 1, \operatorname{ord}_{p} f \geq k\right\} .
$$

Note that $K_{\Omega}^{(0)}(z ; X)$ does not depend on $X$ and is equal to the Bergman kernel $K_{\Omega}(z)$. For $z \in \Omega$ such that $K_{\Omega}(z)>0$ we also have the equality

$$
\beta_{\Omega}^{2}(z ; X)=\frac{K_{\Omega}^{(1)}(z ; X)}{K_{\Omega}(z)} .
$$

TheOREM 1. Let $\Omega$ be a pseudoconvex domain in $\mathbb{C}^{n}, p \in \Omega, a>0$ and let $U \subset \Omega$ be a domain such that $A(p, \Omega, a) \subset U$.

Then for any $k=0,1, \ldots$ there is a constant $C=C(n, k, a)$ such that for any $f \in L_{h}^{2}(U)$ there is a function $F \in \mathcal{O}(\Omega)$ with $F^{(j)}(p)=f^{(j)}(p)$, $j=0,1, \ldots, k$ and $\|F\|_{\Omega} \leq C\|f\|_{A(p, \Omega, a)}$.

In particular, the following localization properties for Bergman kernel and metric hold. There is a constant $C$ (depending only on dimension $n$ and $a$ ) such that

$$
\begin{gathered}
K_{\Omega}(p) \leq K_{U}(p) \leq C K_{\Omega}(p), \\
\frac{1}{C} \beta_{\Omega}(p ; X) \leq \beta_{U}(p ; X) \leq C \beta_{\Omega}(p ; X), \quad X \in \mathbb{C}^{n} .
\end{gathered}
$$

Proof. Let $\left(\Omega_{\nu}\right)$ be a sequence of hyperconvex domains such that $p \in$ $\Omega_{\nu}, \Omega_{\nu} \subset \subset \Omega_{\nu+1}$ and $\bigcup_{\nu=1}^{\infty} \Omega_{\nu}=\Omega$. 
The continuity of $g_{\Omega_{\nu}}(p, \cdot)$ and the convergence $\lim _{z \rightarrow \partial \Omega_{\nu}} g_{\Omega_{\nu}}(p, z)=0$ imply that

$$
A\left(p, \Omega_{\nu}, a\right) \subset \subset U \cap \Omega_{\nu} .
$$

Let $\chi: \mathbb{R} \mapsto \mathbb{R}$ be a $C^{\infty}$-function such that $0 \leq \chi \leq 1,\left|\chi^{\prime}(t)\right| \leq \frac{2}{\log 2}$ and

$$
\chi(t)= \begin{cases}1 & t \geq \log (2 a) \\ 0 & t \leq \log a\end{cases}
$$

Denote also

$$
\phi_{\nu}(\cdot):=2(n+k) g_{\Omega_{\nu}}(p, \cdot), \quad v_{\nu}(\cdot):=g_{\Omega_{\nu}}(p, \cdot) .
$$

Note that

$$
\begin{gathered}
\operatorname{supp} \chi\left(\log \left(-v_{\nu}\right)\right) \subset A\left(p, \Omega_{\nu}, a\right) \subset \subset U \cap \Omega_{\nu}, \\
\operatorname{supp} \chi^{\prime}\left(\log \left(-v_{\nu}\right)\right) \subset\left\{z \in \Omega_{\nu}: g_{\Omega_{\nu}}(p, z) \geq-2 a\right\} \cap A\left(p, \Omega_{\nu}, a\right) \subset \subset U \cap \Omega_{\nu} .
\end{gathered}
$$

Then we may apply Theorem 3.3 in [Bło 2004] to get a function $F_{\nu} \in \mathcal{O}\left(\Omega_{\nu}\right)$ such that

$$
\begin{aligned}
& \int_{\Omega_{\nu}}\left|F_{\nu}-f \chi\left(\log \left(-v_{\nu}\right)\right)\right|^{2} \leq \int_{\Omega_{\nu}}\left|F_{\nu}-f \chi\left(\log \left(-v_{\nu}\right)\right)\right|^{2} e^{-\phi_{\nu}} \\
& \quad \leq 16 \int_{U}\left|f \chi^{\prime}\left(\log \left(-v_{\nu}\right)\right)\right|^{2} e^{-\phi_{\nu}} \leq \frac{4 \cdot 16 e^{4(n+k) a}}{(\log 2)^{2}} \int_{A\left(p, \Omega_{\nu}, a\right)}|f|^{2}<\infty .
\end{aligned}
$$

Since $g_{\Omega \nu}(p, \cdot)-\log \|\cdot-p\|$ is bounded near $p$, we get that $F_{\nu}^{(j)}(p)=f^{(j)}(p)$, $j=0, \ldots, k$.

The above estimates give also the following

$$
\begin{aligned}
\left\|F_{\nu}\right\|_{\Omega_{\nu}} & \leq\left\|F_{\nu}-f \chi\left(\log \left(-v_{\nu}\right)\right)\right\|_{\Omega_{\nu}}+\left\|f \chi\left(\log \left(-v_{\nu}\right)\right)\right\|_{U} \\
& \leq C^{\prime}\|f\|_{A\left(p, \Omega_{\nu}, a\right)}+\|f\|_{A\left(p, \Omega_{\nu}, a\right)} \leq C\|f\|_{A(p, \Omega, a)}
\end{aligned}
$$

where constant $C$ depends only on $n, k$ and $a$. Now one may easily find a function $F \in \mathcal{O}(\Omega)$ such that $F^{(j)}(p)=f^{(j)}(p), j=0, \ldots, k$ and

$$
\|F\|_{\Omega} \leq C\|f\|_{A(p, \Omega, a)},
$$

which completes the proof. 
We formulate below a result which is essentially contained in [Bło 2004] as Theorem 4.1. The formulation in [Bło 2004] is with $a=1$ and for $D$ bounded. Nevertheless, the proof for $a$ arbitrary and bounded $D$ follows exactly the same idea. The case of unbounded $D$ follows from the bounded case by the standard approximation process.

Theorem 2. (see [Bło 2004], Theorem 4.1) Let D be a pseudoconvex domain in $\mathbb{C}^{n}, f \in L_{h}^{2}(D)$ and $a>0$. Then there is a constant $C=C(n, a)$ such that

$$
|f(z)| \leq C\|f\|_{A(z, D, a)} \sqrt{K_{D}(z)}, \quad z \in D .
$$

Note that in view of the Kobayashi criterion (see [Kob 1962]) Theorem 2 implies the following sufficient condition for Bergman completeness of domains (compare [Her 1999] for the bounded case and [Chen-Zhang 2002] for similar result in the unbounded case).

TheOrem 3. Let $a>0$, let $D$ be a pseudoconvex domain in $\mathbb{C}^{n}$ admitting a Bergman metric. Assume that for any sequence of points $\left(z_{\nu}\right) \subset D$ with no accumulation point in $D$ the convergence $\operatorname{Vol}\left(A\left(z_{\nu}, D, a\right)\right)_{\nu \rightarrow \infty} \rightarrow 0$ holds. Then the domain $D$ is Bergman complete.

Below we formulate a result on Bergman exhaustiveness of some bounded domains. The proof contains properties expressed with the help of the function $\gamma_{D}$ defined on an open set $D \subset \mathbb{C}$ (see [Zwo 2002] and [Pfl-Zwo 2003], where the functions considered there are identical up to a negligible difference at the upper limit of the integral). We recall the definition below

$$
\gamma_{D}(z):=\int_{0}^{1 / 4} \frac{d \delta}{\delta^{3}(-\log (\operatorname{cap}(\{w \in \mathbb{C}:|w-z| \leq \delta, w \notin D\})))}, \quad z \in \bar{D},
$$

where 'cap' denotes the logarithmic capacity.

TheOREm 4. Let $D$ be a pseudoconvex domain in $\mathbb{C}^{n}$, and let $u \in$ $\operatorname{PSH}(D)$. For $c \in \mathbb{R}$ define

$$
D_{c}:=\{z \in D: u(z)<c\} .
$$

Assume that $D_{c}$ is bounded. Then

$$
\lim _{D_{c} \ni z \rightarrow z_{0}} K_{D_{c}}(z)=\infty
$$


for any $z_{0} \in \partial D_{c} \cap D$.

Moreover, the following estimate holds: for any $\epsilon>0$ there is a constant $M>0$ such that

$$
K_{D_{c}}(z) \geq \frac{M}{\left(\operatorname{dist}\left(z, \partial D_{c}\right)\right)^{2-\epsilon}}
$$

as $z \rightarrow z_{0}$.

Proof. Let us fix $c \in \mathbb{R}$ and $z_{0} \in \partial D_{c} \cap D$. Then there is an $r_{0} \in\left(0, \frac{1}{4}\right)$ such that $B\left(z_{0}, r\right) \subset D$. Certainly, $u\left(z_{0}\right) \geq c$.

Let $z \in \bar{D}_{c} \cap B\left(z_{0}, r_{0}\right)$. Denote by $L_{z}$ the complex line joining $z_{0}$ and $z$. We identify the subsets of $L_{z}$ with the subsets of $\mathbb{C}$.

We claim that for any $\rho \in\left(\left\|z-z_{0}\right\|, r_{0}-\left\|z-z_{0}\right\|\right)$

$$
\text { there is a } w \in L_{z} \cap\{\|w-z\|=\rho\} \text { such that } u(w) \geq c .
$$

The last condition implies, in particular, that $w \in L_{z} \backslash D_{c}$.

For the proof of (1) suppose that there is a $\rho \in\left(\left\|z-z_{0}\right\|, r_{0}-\left\|z-z_{0}\right\|\right)$ such that for any $w \in L_{z} \cap\{\|w-z\|=\rho\}$ the inequality $u(w)<c$ holds. Then the maximum principle of subharmonic functions and their uppersemicontinuity imply that

$$
u\left(z_{0}\right) \leq \sup \left\{u(w): w \in L_{z},\|w-z\|=\rho\right\}<c \leq u\left(z_{0}\right)
$$

- contradiction.

The properties of the logarithmic capacity imply that for any $\rho \in(\| z-$ $\left.z_{0}\left\|, r_{0}-\right\| z-z_{0} \|\right)$

$$
\operatorname{cap}\left(\left\{w \in L_{z} \backslash D_{c}:\|w-z\| \leq \rho\right\}\right) \geq \frac{\rho-\left\|z-z_{0}\right\|}{4} .
$$

It follows from [Zwo 2002], [Pfl-Zwo 2003] that there is a constant $C>0$ (depending on the diameter of $D_{c}$ and independent of $z_{0}, z$ ) such that (formally the inequalities are formulated for domains; nevertheless, they remain true also for open sets - the sets $D_{c} \cap L_{z}$ need not be connected)

$$
\begin{aligned}
& K_{D_{c} \cap L_{z}}(z) \geq C \gamma_{D_{c} \cap L_{z}}(z) \\
& \quad \geq C \int_{\left\|z-z_{0}\right\|}^{r_{0}-\left\|z-z_{0}\right\|} \frac{d \delta}{\delta^{3}\left(-\log \left(\operatorname{cap}\left(\left\{w \in L_{z} \backslash D_{c}:\|w-z\| \leq \delta\right\}\right)\right)\right)} \\
& \quad \geq C \int_{\left\|z-z_{0}\right\|}^{r_{0}-\left\|z-z_{0}\right\|} \frac{d \delta}{\delta^{3}\left(-\log \left(\frac{\delta-\left\|z-z_{0}\right\|}{4}\right)\right)} .
\end{aligned}
$$


Moreover, the Ohsawa-Takegoshi extension theorem (see [Ohs-Tak 1987]) implies that there is a constant $\tilde{C}>0$ (depending only on the diameter of $D_{c}$ ) such that for any $z \in D_{c}$

$$
K_{D_{c}}(z) \geq \tilde{C} K_{D_{c} \cap L_{z}}(z) .
$$

Consequently,

$$
K_{D_{c}}(z) \geq C \tilde{C} K_{D_{c} \cap L_{z}}(z) \geq C \tilde{C} \int_{\left\|z-z_{0}\right\|}^{r_{0}-\left\|z-z_{0}\right\|} \frac{d \delta}{\delta^{3}\left(-\log \left(\frac{\delta-\left\|z-z_{0}\right\|}{4}\right)\right)},
$$

which implies both claims of the theorem.

Remark. The above theorem is actually a local one, i.e. the estimates given in the theorem hold at such points where the domain is locally given as a sublevel set of a plurisubharmonic function.

Note that the condition (1) implies, in particular, that the set $D_{c} \cap L_{z}$ is regular with respect to the Dirichlet problem at all points of $\partial D_{c} \cap L_{z} \cap D$. This implies, for example, that for any exhausting $u \in \mathrm{SH}(\mathbb{C})$ and for any $c \in \mathbb{R}$ the set $\{u<c\}$ is hyperconvex. The examples of exhausting discontinuous Minkowski functionals in $\mathbb{C}^{n}, n \geq 2$, show that the analogous result does not hold in higher dimension. The other examples of bounded hyperconvex domains in $\mathbb{C}$ are domains $D_{c}$ with $D$ being bounded and hyperconvex.

Below we formulate as a corollary of Theorem 4 a result which intersects some known results on Bergman exhaustiveness of domains (see e.g. [Ohs 1993], [Jar-Pfl-Zwo 2000]).

Corollary 5. (1) Let D be a strictly hyperconvex or a bounded balanced pseudoconvex domain. Then $D$ is Bergman exhaustive.

(2) Let $D$ be a bounded pseudoconvex Hartogs domain over the base $\Omega$ in $\mathbb{C}^{n}$. Assume that $\tilde{z}^{0}=\left(z^{0}, z_{n+1}^{0}\right) \in \partial D$, where $z^{0} \in \Omega$. Then

$$
\lim _{D \ni\left(z, z_{n+1}\right) \rightarrow\left(z^{0}, z_{n+1}^{0}\right)} K_{D}\left(z, z_{n+1}\right)=\infty .
$$

In particular, if $\Omega$ is Bergman exhaustive then so is $D$.

Now we formulate some results on Bergman completeness of a class of unbounded domains. In the unbounded case we have to overcome additional, in comparison with the bounded one, difficulties. Namely, posi- 
tivity of the Bergman kernel $K_{D}$, and the existence of the Bergman metric need not hold. The problem of the Bergman completeness in the unbounded case was studied, for instance, in [Chen 2004], [Chen-Zhang 2002] and [Chen-Kam-Ohs 2004].

Below we show a new class of unbounded domains admitting the Bergman metric, which are Bergman exhaustive and Bergman complete. The examples we deliver are some Hartogs domains with the base equal to $\mathbb{C}^{n}$.

Let $\rho: \mathbb{C}^{n} \mapsto \mathbb{R}$ be a plurisubharmonic function which is bounded from below. Define

$$
D_{\rho}:=\left\{\tilde{z}=\left(z, z_{n+1}\right) \in \mathbb{C}^{n} \times \mathbb{C}:\left|z_{n+1}\right|<e^{-\rho(z)}\right\} .
$$

Note that $D_{\rho}$ is a pseudoconvex Hartogs domain, which is unbounded and not hyperconvex - note for example that $\mathbb{C}^{n} \times\{0\} \subset D_{\rho}$.

Note that the space

$$
\mathcal{E}:=\operatorname{Span}\left\{f(z) z_{n+1}^{l}: f \in \mathcal{O}\left(\mathbb{C}^{n}\right), l \in \mathbb{Z}_{+}: f(z) z_{n+1}^{l} \in L_{h}^{2}\left(D_{\rho}\right)\right\}
$$

is dense in $L_{h}^{2}\left(D_{\rho}\right)$ - use the expansion in the Hartogs series of any function from $L_{h}^{2}\left(D_{\rho}\right)$.

Theorem 6. Let $\rho: \mathbb{C} \mapsto \mathbb{R}$ be as above and assume additionally that

$$
\lim _{\|z\| \rightarrow \infty}\|z\|^{k} e^{-\rho(z)}=0 \quad \text { for any } k>0 .
$$

Then the domain $D_{\rho}$ admits a Bergman metric and is Bergman exhaustive and Bergman complete.

Remarks. Note that the condition (3) is equivalent to the property

$$
\lim _{\|z\| \rightarrow \infty}\left|z^{\alpha}\right| e^{-\rho(z)}=0 \quad \text { for any } \alpha \in \mathbb{Z}_{+}^{n}
$$

or to the property

$$
\lim _{\left|z_{j}\right| \rightarrow \infty}\left|z_{j}^{k}\right| e^{-\rho(z)}=0 \quad \text { for any } j=1, \ldots, n, k \in \mathbb{Z}_{+} \cdot
$$

For any $k \in \mathbb{Z}_{+}, j=1, \ldots, n$ define $M_{k, j}:=\sup \left\{\left|z_{n+1}\right|\left|z_{j}\right|^{k}:\left(z^{\prime}, z_{n+1}\right) \in\right.$ $\left.D_{\rho}\right\}<\infty$. Then the set

$$
\tilde{D}:=\left\{\tilde{z}=\left(z, z_{n+1}\right) \in \mathbb{C}^{n+1}:\left|z_{n+1}\right|\left|z_{j}\right|^{k}<M_{k, j}, j=1, \ldots, n, k \in \mathbb{Z}_{+}\right\}
$$


is a pseudoconvex Reinhardt domain such that $D_{\rho} \subset \tilde{D}$ and

$$
\mathfrak{C}(\tilde{D})=(-\infty, 0]^{n+1} .
$$

Let us recall (see [Zwo 1999] and [Zwo 2000]) that for the Reinhardt pseudoconvex domain $D \subset \mathbb{C}^{n}$ the set

$$
\mathfrak{C}(D):=\left\{v \in \mathbb{R}^{n}: a+\mathbb{R}_{+} v \subset \log D\right\},
$$

where $a \in \log D$ is some (any) point from $\log D$ - the logarithmic image of $D$ - is a closed convex cone.

We may also define

$$
\tilde{\rho}(z):=\max \left\{\rho\left(e^{i \theta_{1}} z_{1}, \ldots, e^{i \theta_{n}} z_{n}\right): \theta_{1}, \ldots, \theta_{n} \in \mathbb{R}\right\}, \quad z \in \mathbb{C}^{n} .
$$

Then $\tilde{\rho}$ is a plurisubharmonic function on $\mathbb{C}^{n}, \rho \leq \tilde{\rho}$ and $\tilde{\rho}$ is invariant with respect to rotations of coordinates. Obviously, $D_{\tilde{\rho}} \subset D_{\rho}$.

Proof. Equality (4) implies that for any $\alpha \in \mathbb{Z}_{+}^{n}, l \in \mathbb{Z}_{+}$the functions $z^{\alpha} z_{n+1}^{l} \in L_{h}^{2}(\tilde{D})$ (see [Zwo 1999], [Zwo 2000]), so $z^{\alpha} z_{n+1}^{l} \in L_{h}^{2}\left(D_{\rho}\right)$. In particular, $K_{D_{\rho}}>0$ on $D_{\rho}$; moreover, $D_{\rho}$ admits a Bergman metric.

Moreover,

$$
\lim _{\left(z, z_{n+1}\right) \in D_{\rho}:\left\|\left(z, z_{n+1}\right)\right\| \rightarrow \infty} K_{D_{\rho}}\left(z, z_{n+1}\right)=\infty
$$

(use the fact that $z_{j} \in L_{h}^{2}\left(D_{\rho}\right), j=1, \ldots, n$ ).

Let $\left(z^{0}, z_{n+1}^{0}\right) \in \partial D_{\rho}$ and $\left(z^{\nu}, z_{n+1}^{\nu}\right) \rightarrow\left(z^{0}, z_{n+1}^{0}\right)$. Consider the biholomorphic mapping

$$
\Phi: \mathbb{C}_{*}^{n} \times \mathbb{C} \ni\left(z, z_{n+1}\right) \longmapsto\left(z_{1} z_{n+1}, \ldots, z_{n} z_{n+1}, z_{n+1}\right) \in \mathbb{C}_{*}^{n} \times \mathbb{C} .
$$

Note that $\operatorname{det} \Phi^{\prime}\left(z^{0}, z_{n+1}^{0}\right) \neq 0, \mathbb{C}^{n} \times\{0\}$ is removable for $L_{h}^{2}$-functions and $\Phi\left(D_{\rho} \cap\left(\mathbb{C}^{n} \times \mathbb{C}_{*}\right)\right)=\left\{\left(w, w_{n+1}\right) \in \mathbb{C}^{n} \times \mathbb{C}_{*}: \log \left|w_{n+1}\right|+\rho\left(\frac{w_{1}}{w_{n+1}}, \ldots, \frac{w_{n}}{w_{n+1}}\right)<\right.$ $0\}$ is bounded and Bergman exhaustive at $\Phi\left(z_{1}^{0}, z_{2}^{0}\right)$ (use Theorem 4). Consequently, the formula for the behaviour of the Bergman kernel under biholomorphic mappings implies $\lim _{\nu \rightarrow \infty} K_{D_{\rho}}\left(z^{\nu}, z_{n+1}^{\nu}\right)=\infty$. Consequently, we finished the proof of Bergman exhaustiveness of $D_{\rho}$.

To finish the proof of the completeness of $D_{\rho}$ we verify that the Kobayashi criterion is satisfied at every boundary point (including $\infty$ ) for the space $\mathcal{E}$. 
First note that the functions $f(z) z_{n+1}^{l}$, where $f \in \mathcal{O}\left(\mathbb{C}^{n}\right)$ and $f(z) z_{n+1}^{l} \in$ $L_{h}^{2}\left(D_{\rho}\right)$, are bounded near $\left(z^{0}, z_{n+1}^{0}\right)$ for any $\left(z^{0}, z_{n+1}^{0}\right) \in \partial D_{\rho}$. This finishes the proof in the case of finite boundary points. What remains is to verify the Kobayashi criterion for sequences accumulating at infinity.

To verify the Kobayashi criterion at infinity we prove the following property

$$
\lim _{D_{\rho} \ni\left(p, p_{n+1}\right):\left|p_{1}\right| \rightarrow \infty} g_{D_{\rho}}\left(\left(p, p_{n+1}\right),\left(z, z_{n+1}\right)\right)=0
$$

for any $\left(z, z_{n+1}\right) \in D_{\rho} \cap\left(\mathbb{C} \times \mathbb{C}_{*}\right)$.

Since $\operatorname{Vol}\left(D_{\rho}\right)<\infty$ this implies in particular that

$$
\operatorname{Vol}\left(A\left(\left(p, p_{n+1}\right), D, 1\right)\right) \rightarrow 0
$$

as $\left\|\left(p, p_{n+1}\right)\right\| \rightarrow \infty$. Consequently, because of Theorem 2, this property shows that the Kobayashi criterion is satisfied at infinity, too.

Below we show (5).

Without loss of generality we may assume that $D_{\rho} \subset \mathbb{C}^{n} \times \mathbb{D}$. Recall the definition

$$
M_{k, j}:=\sup \left\{\left|z_{n+1}\right|\left|z_{j}\right|^{k}:\left(z, z_{n+1}\right) \in D_{\rho}\right\}<\infty, \quad j=1, \ldots, n, k \in \mathbb{Z}_{+} .
$$

Now fix $k=1,2, \ldots$ and $j=1, \ldots, n$ for a while. Let $\left(p, p_{n+1}\right) \in D_{\rho}$. For $\left(z, z_{n+1}\right) \in D_{\rho}$ with $\left|z_{j}\right| \leq 2\left|p_{j}\right|$ we get

$$
\left|\left(z_{j}-p_{j}\right)^{k} z_{n+1}\right| \leq 3^{k}\left|p_{j}\right|^{k} .
$$

For $\left(z, z_{n+1}\right) \in D_{\rho}$ with $\left|z_{j}\right|>2\left|p_{j}\right|$ we get

$$
\left|\left(z_{j}-p_{j}\right)^{k} z_{n+1}\right| \leq\left(\frac{3}{2}\right)^{k}\left|z_{j}\right|^{k}\left|z_{n+1}\right| \leq\left(\frac{3}{2}\right)^{k} M_{k, j} .
$$

Consequently,

$$
\left|\left(z_{j}-p_{j}\right)^{k} z_{n+1}\right| \leq 3^{k}\left|p_{j}\right|^{k}+\left(\frac{3}{2}\right)^{k} M_{k, j}, \quad\left(z, z_{n+1}\right) \in D_{\rho} .
$$

Since the right side in the formula below is a competitor in the definition of $g_{D_{\rho}}\left(\left(p, p_{n+1}\right), \cdot\right)$, we get the inequality

(6) $g_{D_{\rho}}\left(\left(p, p_{n+1}\right),\left(z, z_{n+1}\right)\right) \geq \log \sqrt[k]{\frac{\left|\left(z_{j}-p_{j}\right)^{k} z_{n+1}\right|}{3^{k}\left|p_{j}\right|^{k}+\left(\frac{3}{2}\right)^{k} M_{k, j}}}, \quad\left(z, z_{n+1}\right) \in D_{\rho}$. 
The above formula will be fundamental for further computations (note that the formula holds for arbitrary $\left(p, p_{n+1}\right), k, j$ and $\left.\left(z, z_{n+1}\right)\right)$.

Fix $r>0$ for a while. The set $\left\{\left(z, z_{n+1}\right) \in D_{\rho}:\left|z_{n+1}\right| \geq r\right\}$ is bounded. Letting $\left|p_{j}\right| \rightarrow \infty$ we get that for any $k$ there is a $\delta_{k, j}>0$ such that for any $\left(p, p_{n+1}\right) \in D_{\rho}$ with $\left|p_{j}\right| \geq \delta_{k, j}$

$$
g_{D_{\rho}}\left(\left(p, p_{n+1}\right),\left(z, z_{n+1}\right)\right) \geq 2 \log \frac{\sqrt[k]{r}}{3}, \quad\left(z, z_{n+1}\right) \in D_{\rho},\left|z_{n+1}\right| \geq r
$$

Then letting $k \rightarrow \infty$ we get the following property: there is an $s>0$ such that for any $\left(p, p_{n+1}\right) \in D_{\rho}$ with $\left|p_{j}\right| \geq s$ the inequality

$$
g_{D_{\rho}}\left(\left(p, p_{n+1}\right),\left(z, z_{n+1}\right)\right) \geq-3 \log 3
$$

holds for any $\left(z, z_{n+1}\right) \in D_{\rho}$ with $\left|z_{n+1}\right| \geq r$.

It follows from the definition of $g_{D_{\rho}}\left(\left(p, p_{n+1}\right), \cdot\right)$ that

$$
g_{D_{\rho}}\left(\left(p, p_{n+1}\right),\left(z, z_{n+1}\right)\right) \geq \frac{-4 \log 3 \log \frac{\left|z_{n+1}-p_{n+1}\right|}{\left|1-\bar{p}_{n+1} z_{n+1}\right|}}{\log r}
$$

for any $\left(p, p_{n+1}\right),\left(z, z_{n+1}\right) \in D_{\rho}$ with $\left|p_{j}\right| \gg s$ and $\left|z_{n+1}\right| \geq r$.

Now fix $\left(z, z_{n+1}\right) \in D_{\rho} \cap\left(\mathbb{C} \times \mathbb{C}_{*}\right)$ then we easily finish the proof making use of the last property. Namely, we let $r \rightarrow 0$ and then (simultaneously) $\max \left\{\left|p_{j}\right|: j=1, \ldots, n\right\} \rightarrow \infty$.

Below we present a result showing that the assumption (3) in Theorem 6 is essential.

THEOREM 7. Let $\rho: \mathbb{C} \mapsto \mathbb{R}$ be a subharmonic function bounded from below. Assume that there is a $k>0$ such that $\liminf _{|\lambda| \rightarrow \infty}|\lambda|^{k} e^{-\rho(\lambda)}>0$ and that $D_{\rho}$ admits a Bergman metric. Then the domain $D_{\rho} \subset \mathbb{C}^{2}$ is not Bergman complete.

In particular, if $\rho(\lambda):=k \log |\lambda|, k \gg 1$, then $D_{\rho}$ has a Bergman metric, but it is not Bergman complete.

Remark. Under additional assumption that $D_{\rho}$ is Reinhardt, i.e. $\rho$ is radial, the above result is contained in [Juc 2004].

Proof. Without loss of generality we may assume that

$$
\left\{\left(z_{1}, z_{2}\right) \in \mathbb{C}^{2}:\left|z_{2}\right|<1,\left|z_{1}^{k} z_{2}\right|<1\right\} \subset D_{\rho}
$$


where $k$ is a positive integer.

Consider the biholomorphic mapping

$$
\Phi: \mathbb{C}_{*}^{2} \ni\left(z_{1}, z_{2}\right) \longmapsto\left(z_{1}^{k+1} z_{2}, z_{1}^{k} z_{2}\right) \in \mathbb{C}_{*}^{2} .
$$

Note that

$$
\left\{\left(w_{1}, w_{2}\right) \in \mathbb{C}_{*}^{2}:\left|w_{2}\right|^{k+1}<\left|w_{1}\right|^{k},\left|w_{2}\right|<1\right\} \subset \Phi\left(D_{\rho} \cap \mathbb{C}_{*}^{2}\right) .
$$

Since the sequence $\left(\left(\frac{1}{2}, \frac{1}{\nu}\right)\right)_{\nu}$ is a Cauchy sequence for the Bergman distance in $\Phi\left(D_{\rho} \cap \mathbb{C}_{*}^{2}\right)$, the sequence $\left(\frac{\nu}{2}, \frac{2^{k}}{\nu^{k+1}}\right)=\Phi^{-1}\left(\frac{1}{2}, \frac{1}{\nu}\right)$ is a Cauchy sequence for the Bergman distance in $D_{\rho} \cap \mathbb{C}_{*}^{2}$ and therefore it is a Cauchy sequence for the Bergman distance in $D_{\rho}$, which has no accumulation point in $D_{\rho}$.

\section{REFERENCES}

[Bło 2004]

[Bło-Pfl 1998]

[Bre 1955]

[Chen 2004]

[Chen-Zhang 2002]

[Chen-Zhang 2004]

[Chen-Kam-Ohs 2004]

[Her 1999]

[Jar-Pfl 1993]

[Jar-Pfl-Zwo 2000]

[Juc 2004]

[Kli 1991]
Z. Błocki, The Bergman metric and the pluricomplex Green function, Trans. Amer. Math. Soc., 357 (2005), 2613-2625.

Z. Błocki and P. Pflug, Hyperconvexity and Bergman completeness, Nagoya Math. J., 151 (1998), 221-225.

J. Bremermann, Holomorphic continuation of the kernel and the Bergman metric, Lectures on Functions of a Complex Variable, Univ. of Michigan Press (1955), pp. 349-383.

B.-Y. Chen, Bergman completeness of hyperconvex manifolds, Nagoya Math. J., 175 (2004), 165-170.

B.-Y. Chen and J.-H. Zhang, The Bergman metric on a Stein manifold with a bounded plurisubharmonic function, Trans. Amer. Math. Soc., 354 (2002), 2997-3009.

B.-Y. Chen and J.-H. Zhang, Addendum to 'The Serre problem on certain bounded domains', preprint.

B.-Y. Chen, J. Kamimoto and T. Ohsawa, Behavior of the Bergman kernel at infinity, Math. Z., 248 (2004), 695-708.

G. Herbort, The Bergman metric on hyperconvex domains, Math. Z., 232 (1999), 183-196.

M. Jarnicki and P. Pflug, Invariant Distances and Metrics in Complex Analysis, Walter de Gruyter, vol. 8, 1993.

M. Jarnicki, P. Pflug and W. Zwonek, On Bergman completeness of non-hyperconvex domains, Univ. Iag. Acta Math., 38 (2000), 169-184.

P. Jucha, Bergman functions in $\mathbb{C}$ and $\mathbb{C}^{n}$, Dissertation (in Polish) (2004).

M. Klimek, Pluripotential Theory, Oxford University Press, 1991. 
[Kob 1962] S. Kobayashi, On complete Bergman metrics, Proc. Amer. Math. Soc., 13 (1962), 511-513.

[Ohs 1993] T. Ohsawa, On the Bergman kernel of hyperconvex domains, Nagoya Math. J., 129 (1993), 43-52.

[Ohs-Tak 1987] T. Ohsawa and K. Takegoshi, On the extension of $L^{2}$-holomorphic functions, Math. Z., 195 (1987), 197-204.

[Pfl-Zwo 2003] P. Pflug and W. Zwonek, Logarithmic capacity and Bergman functions, Arch. Math. (Basel), 80 (2003), 536-552.

[Ran 1995] T. Ransford, Potential Theory in the Complex Plane, Cambridge University Press, 1995.

[Zwo 1999] W. Zwonek, On Bergman completeness of pseudoconvex Reinhardt domains, Ann. Fac. Sci. Toulouse, 8 (1999), 537-552.

[Zwo 2000] W. Zwonek, Completeness, Reinhardt domains and the method of complex geodesics in the theory of invariant functions, Diss. Math., 388 (2000), 1-103.

[Zwo 2002] W. Zwonek, Wiener's type criterion for Bergman exhaustiveness, Bull. Pol. Acad.: Math., 50 (2002), 297-311.

Peter Pflug

Carl von Ossietzky Universität Oldenburg

Institut für Mathematik

Postfach 2503

D-26111 Oldenburg

Germany

pflug@mathematik.uni-oldenburg.de

Włodzimierz Zwonek

Uniwersytet Jagielloński

Instytut Matematyki

Reymonta 4

30-059 Kraków

Poland

Wlodzimierz.Zwonek@im.uj . edu.pl 